Noordman, J., Vet, E. de, Weijden, T. van der, Dulmen, S. van. Motivational interviewing within the different stages of change: an analysis of practice nurse-patient consultations aimed at promoting a healthier lifestyle. Social Science \& Medicine: 2013, 87, 60-67

\begin{tabular}{|l|l|}
\hline $\begin{array}{l}\text { Postprint } \\
\text { Version }\end{array}$ & 1.0 \\
\hline Journal website & http://www.sciencedirect.com/science/article/pii/S0277953613001810 \\
\hline Pubmed link & $\underline{\text { http://www.ncbi.nlm.nih.gov/pubmed/23631779 }}$ \\
\hline DOI & $10.1016 /$ j.socscimed.2013.03.019 \\
\hline
\end{tabular}

This is a NIVEL certified Post Print, more info at http://www.nivel.eu

\title{
Motivational interviewing within the different stages of change: An analysis of practice nurse- patient consultations aimed at promoting a healthier lifestyle
}

\author{
JANNEKE NOORDMAN ${ }^{\mathrm{A},}$ EMELY DE VET ${ }^{\mathrm{B}}$, TRUDY VAN DER WEIJDEN ${ }^{\mathrm{C}}$, SANDRA VAN \\ DULMEN $^{\mathrm{A}, \mathrm{D}, \mathrm{E}}$ \\ a NIVEL (Netherlands Institute for Health Services Research), PO Box 1568, 3500 BN \\ Utrecht, The Netherlands \\ ${ }^{b}$ Utrecht University, Clinical and Health Psychology, P.O. Box 80140, 3508 TC Utrecht, The \\ Netherlands \\ ${ }^{\mathrm{c}}$ Maastricht University, School for Public Health and Primary Care (CAPHRI), Department of \\ General Practice, P.O. Box 616, 6200 MD Maastricht, The Netherlands \\ ${ }^{d}$ Radboud University Nijmegen Medical Centre, Department of Primary and Community \\ Care, Geert Grooteplein Noord 21, 6500 HB Nijmegen, The Netherlands \\ ${ }^{\mathrm{e}}$ Department of Health Science, Buskerud University College, Drammen, Norway
}

\begin{abstract}
Combining the Stages of Change (SOC) model with Motivational Interviewing (MI) is seen as a helpful strategy for health care providers to guide patients in changing unhealthy lifestyle behaviour. SOC suggests that people are at different stages of motivational readiness for engaging in health behaviours and that intervention methods are most useful when tailored to a person's stage of change. However, it is unknown whether practice nurses (PNs) actually adapt their MI and more generic communication skills to a particular stage during real-life face-to-face consultations with their patients. The aim of this study was to explore whether and how PNs apply MI and general communication skills to the different SOC of patients, targeting behaviour change about smoking, alcohol use, dietary habits and/or physical activity.

Real-life consultations between nineteen Dutch PNs and 103 patients were recorded on video between June 2010 and March 2011. All consultations focused on a discussion of patients' lifestyle behaviour. The Behaviour Change Counselling Index (BECCI) was used to code PNs' MI skills. Generic communication skills were rated with the MAAS-global. Patients' SOC was assessed for each consultation by observing the communication between patient and PN regarding the patient's current lifestyle behaviour.
\end{abstract}


Noordman, J., Vet, E. de, Weijden, T. van der, Dulmen, S. van. Motivational interviewing within the different stages of change: an analysis of practice nurse-patient consultations aimed at promoting a healthier lifestyle. Social Science \& Medicine: 2013, 87, 60-67

Multilevel analyses revealed that PNs adapt their MI skills to a patient's SOC to some extent. On average PNs apply MI skills more to patients in the preparation stage $(\mathrm{P}<0.05)$ than during the other stages of change. PNs adjusted three MI skills and one generic communication skill to patients' SOC. This explorative study suggests that, at least to some extent, PNs intuitively assess the stage of patients' readiness to change and tailor their communication accordingly. However, differences between the stages were small. By teaching PNs to explicitly identify patients' SOC they could further enhance and adapt their MI and general communication skills to the individual.

\section{INTRODUCTION}

Primary health care is seen as an appropriate setting for the prevention and management of a patient's lifestyle behaviour (Coups, Gaba, \& Orleans, 2004; Nielen et al., 2010; WHO, 2002). In primary care, addressing lifestyle behaviour and referring to programmes promoting lifestyle can generally be carried out by a practice nurse (PN). Previous studies indeed show that PNs adequately monitor patients with chronic conditions, provide patients with lifestyle advice and guide them during smoking cessation and weight reduction (Laurant et al., 2009; Richards, Carley, Jenkins-Clarke, \& Richards, 2000).

Most primary care providers rely on their (authoritative) professional role to convince people to change by providing information or advice about behaviour change, although the effectiveness of these methods are ambiguous (Britt, Hudson, \& Blampied, 2004; Davis et al., 2011; Elder, Ayala, \& Harris, 1999; Miller \& Rollnick, 2004). Such a traditional persuasive approach does not do justice to the complex nature of changing lifestyle behaviour (i.e. smoking, alcohol use, dietary habits or physical inactivity). Health behaviour change requires effort, motivation and time from both patients and health care providers. At the same time, patients are often ambivalent about behaviour change (Britt et al., 2004; Miller, 1996) and have variable levels of motivation over the course of time (Lange \& Tigges, 2005). A more promising approach to changing unhealthy behaviour is motivational interviewing (MI) (Bowen et al., 2002; Hardcastle, Taylor, Bailey, \& Castle, 2008; Richards, Kattelmann, \& Ren, 2006). MI is a patient-centred directive approach to enhance intrinsic motivation to behavioural change by helping patients explore and resolve ambivalence between the desired behaviour and their actual behaviour (Martins \& McNeil, 2009; Miller \& Rollnick, 2002). It focuses on what patients can do to improve their own health, as opposed to health care providers telling them what to do.

Since patients have varying levels of motivation over time (Lange \& Tigges, 2005), combining MI with the Stages Of Change (SOC) construct from Prochaska \& DiClemente's Transtheoretical Model is seen as a possible potentially helpful strategy to help patients change their behaviour (Emmons \& Rollnick, 2001;Lange \& Tigges, 2005; Miller \& Rollnick, 2004; Petrocelli, 2002). According to the SOC, individuals are at different stages of motivational readiness for engaging in health behaviours, and intervention methods (i.e. interpersonal communication) are most useful when they are tailored to a person's current stage of motivational readiness. The core organizing principle of the Transtheoretical Model is the SOC construct, 
Noordman, J., Vet, E. de, Weijden, T. van der, Dulmen, S. van. Motivational interviewing within the different stages of change: an analysis of practice nurse-patient consultations aimed at promoting a healthier lifestyle. Social Science \& Medicine: 2013, 87, 60-67

which represents a temporal dimension and distinguishes five stages of change indicating individuals' predispositions to change: precontemplation (no intention to change behaviour within the next 6 months), contemplation (intention to change behaviour within the next 6 months, but does not act on intention to change behaviour), preparation (intention to change behaviour within 30 days), action (people changed from unhealthy to healthy behaviour within the past 6 months), and maintenance (maintenance of the behaviour change for more than 6 months) (Elder et al., 1999; Petrocelli, 2002; Prochaska \& DiClemente, 1983;Prochaska, DiClemente, \& Norcross, 1992; Prochaska, Redding, \& Evers, 2002). Progression between the stages is not linear and patients may move forward and backward. Individuals in the various stages differ with respect to the stimuli and barriers they experience in the process of health behaviour change. In other words, the factors and processes hindering or facilitating behaviour change are assumed to differ in each specific stage of change. Hence, patients are assumed to benefit most if health care providers adapt their communication to the individual's SOC (Elder et al., 1999; Petrocelli, 2002; Ruggiero, 2000). However, there are studies that indicate that the Transtheoretical Model or SOC construct is not beneficial in changing behaviour (West, 2005).

Although the idea that communication should be tailored to individuals (stage of change) is widely acknowledged, it remains unknown whether PNs actually adapt their MI and/or more generic communication strategies to a particular SOC during real-life face-to-face consultations with their patients. The aim of the present study is to explore if and to what extent PNs apply MI techniques and general communication skills (including clinical competence) during the different SOC for patients, with emphasis on targeting behaviour change about smoking, alcohol use, dietary habits and/or physical activity.

We hypothesize that PNs are more likely to invite and encourage the patient to talk about behaviour change during a patient's precontemplation and contemplation stage, than during the other SOC. This is also true more for during the preparation stage than during the action and maintenance stage. This can be seen as what is described as 'consciousness raising' (Prochaska et al., 1992), even though theoretically patients in the precontemplation stage benefit most from 'consciousness raising' (Prochaska et al., 1992). In addition, we expect that PNs are more likely 'to ask questions to elicit how the patient thinks and feels about behaviour change' during the precontemplation and contemplation stage than during the other stages. This is reasonably in accordance with the principle of 'dramatic relief' (Prochaska et al., 1992). However, in theory, patients in the precontemplation stage benefit most from 'dramatic relief' (Prochaska et al., 1992). Furthermore, we expect that PNs are more likely 'to acknowledge challenges about behaviour change that the patient faces' during the patient's preparation stage, or during the action and maintenance stage, rather than in the precontemplation or contemplation stage, since these are stages where the patient is ready to change or is already changing. Besides, we hypothesize that PNs and patients are more likely to exchange ideas about how the patient could change current behaviour during the preparation stage than during the SOC, since patients in the preparation stage show willingness to change behaviour. Finally, we 
Noordman, J., Vet, E. de, Weijden, T. van der, Dulmen, S. van. Motivational interviewing within the different stages of change: an analysis of practice nurse-patient consultations aimed at promoting a healthier lifestyle. Social Science \& Medicine: 2013, 87, 60-67

expect that PNs' clinical competence, i.e. their ability to adhere to relevant practice guidelines, will be performed independent of patient's SOC. We had no preconceived ideas concerning the generic communication skills of PNs in relation to patient's SOC since there is no published literature on this topic.

\section{METHOD}

\section{Participants and procedure}

Nineteen Dutch PNs from eight general practices participated in this study and agreed to have approximately ten consecutive, routine consultations videotaped between June 2010 and March 2011. General practices were located throughout The Netherlands. All PNs from seven practices were approached by contacting the GPs from these practices who (except for one practice) had participated in an earlier study (Noordman, Verhaak, van Beljouw, \& van Dulmen, 2010). GPs from one other practice (a health care centre) contacted us for inclusion of all of their PNs. On average PNs had 4.5 years of working experience $(\mathrm{SD}=2.79)$. All nineteen PNs were trained in MI as part of their education, and thirteen of the PNs had undergone extra post-education training in MI (Noordman et al., 2012).

Consultations were video-taped with an unmanned camera located in the consulting room during one or two random days. Adult patients who were scheduled for an appointment with the PN were approached beforehand by a researcher in the waiting room ( $\mathrm{n}=181$, excluding non-response 7.7\%). All participating PNs and patients filled in an informed consent form before recording of the consultation. Participants could withdraw their consent at any time; no one did. Before the recording of the consultation, patients were asked to fill in a questionnaire about their sociodemographic characteristics (e.g. age, gender), the reason for their consultation and their current lifestyle behaviour (smoking, physical activity).

PNs completed a short questionnaire after each recorded consultation. This questionnaire contained information about patients' characteristics (e.g. age, gender) and perceived complaints and diseases. The study was carried out according to Dutch privacy regulations were approved by the Dutch Data Protection Authority.

According to Dutch legislation, approval by a medical ethics committee was not required for this observational study.

\section{Selection of patients and discussion of lifestyle behaviour}

Patients were selected who indicated in the patient questionnaire that they smoked or reported a medium intensive physical activity level of less than 30 min a day, five days a week, according to Dutch guidelines (Kemper, Ooijendijk, \& Stiggelbout, 2000; NNGB, 1998). A second selection criterion was that the lifestyle behaviour (smoking, physical activity, alcohol use or dietary behaviour) of the included patients was discussed during the video-recorded consultation. In total, video-recorded consultations of 149 patients were selected, of which 103 consultations were analysed. Forty-six consultations were not included because lifestyle behaviour was not discussed (even though patients smoked or did not meet the recommended level of physical activity, $\mathrm{n}=16$ ) or because the patient's SOC could not be coded reliably $(\mathrm{n}=30)$.

\section{Video-observations}

The videotaped consultations were rated using two protocols: (1) the MAAS-global (Van Thiel, Ram, \& van Dalen, 2000) and (2) the Behaviour Change Counselling 
Noordman, J., Vet, E. de, Weijden, T. van der, Dulmen, S. van. Motivational interviewing within the different stages of change: an analysis of practice nurse-patient consultations aimed at promoting a healthier lifestyle. Social Science \& Medicine: 2013, 87, 60-67

Index (BECCI) (Lane, 2002; Lane et al., 2005). Observer software (Noldus, Trienes, Hendriksen, Jansen, \& Jansen, 2000) was used to code the video-recorded consultations; items of the observation protocols were incorporated in Observer. The MAAS-global, a validated instrument (Van Thiel et al., 2000), was used to rate PNs' generic communication and clinical competence. This protocol is divided into three sections (see Appendix 1): communication skills for each separate consultation phase (from introduction until evaluation of the consultation), general communication skills (e.g. exploration, information giving) and medical aspects (compliance with guidelines). Each item is to be rated on a scale ranging from 0 'not present' to 6 'excellent'.

The BECCI checklist (Lane, 2002; Lane et al., 2005) was used to code the application of MI for each consultation. The BECCI contains eleven, five-point Likert-scaled items related to the health care providers' behaviours and MI techniques, ranging from 'not at all' to 'a great extent' (see Appendix 2). These items are subdivided into four domains: agenda setting and permission seeking (two items, Cronbach's $\alpha=0.49$ ); the why and how of change in behaviour (five items, $\alpha=0.60$ ); the whole consultation (three items, $\alpha=0.60$ ); and talk about targets (one item). In previous research, BECCI has demonstrated acceptable levels of reliability, validity and sensitivity to detect change (Lane et al., 2005; Moran, Bekker, \& Latchford, 2008; Spollen et al., 2010). Owing to low to moderate Cronbach's alpha scores for the BECCI domains in this study, the eleven separate items of the BECCI were also analysed.

In addition we observed the patient's stage of change (SOC) for each consultation by identifying the patient's current lifestyle behaviour through the communication interaction between patient and PN. The definitions of the five different stages of change were in line with Prochaska \& DiClemente's (1983; Prochaska et al., 1992) SOC model (see introduction). When a patient presented multiple unhealthy behaviours during one consultation we selected one behaviour for which we defined the SOC: (1) the behaviour for the SOC which became most evident during the consultation, and if multiple behaviours still remained (2) the behaviour for which acting upon change was, at that moment, most profitable (e.g. the most earliest SOC level), as indicated during the consultation.

To assess interrater reliability, ten percent of the consultations were rated by two observers independently, resulting in sufficiently high average Kappa scores (Sim \& Wright, 2005) of 0.81 (range 0.72-1.00) for the BECCI, 0.82 (range 0.54-1.00) for the MAAS-global and total agreement for SOC (Kappa score of 1.00).

\section{Statistical analysis}

Firstly, characteristics of PNs, and patients within their stages of change were described and tested with a Chi2 test for categorical variables or an Anova for continuous variables. To explore the interrelationships between all communication items and constructs, we computed Pearson correlation coefficients. We clustered the stages of precontemplation and contemplation into one category, and did the same for action and maintenance, since only few people were in the precontemplation $(n=8)$ and maintenance $(n=3)$ stage. This resulted in three (combined) stages of 
Noordman, J., Vet, E. de, Weijden, T. van der, Dulmen, S. van. Motivational interviewing within the different stages of change: an analysis of practice nurse-patient consultations aimed at promoting a healthier lifestyle. Social Science \& Medicine: 2013, 87, 60-67

change; (1) precontemplation and contemplation ( $\mathrm{n}=47)$, (2) preparation $(\mathrm{n}=22)$, and (3) action and maintenance $(\mathrm{n}=34)$.

Secondly multilevel linear and logistic regression analyses with a random intercept were performed to determine the association between the MAAS-global scores, BECCI domain scores and separate BECCI items (dependent variables) with the different stages of change (model 1).

The multilevel technique was used to correct for clustering of patients within PNs (Snijders \& Bosker, 1999). The categories of SOC (e.g. $0=$ not (pre-)contemplation and $1=($ pre)contemplation) were coded as dummy variables. Thereafter, model 1 was corrected for confounding. Patients' characteristics (age, gender, lifestyle behaviour) were added to the model (model 2). For every dependent variable (e.g. BECCI domain 1) a separate model was created (model 1 and 2) with the different stages of change (e.g. preparation) in order to avoid multiple testing. The analyses were performed in Stata 11 (Stata, 2009) using xtmixed for multilevel linear regression (BECCI domains; BECCI mean sum score; BECCI items 1-5,8-11;

MAAS-global items 2,4-6,8,10-17) and gllamm (Rabe-Hesketh, Skrondal, \& Pickles, 2002) for multilevel logistic regression (BECCI items 6,7; MAAS-global items $1,3,7,9)$. In case of multilevel logistic regression dependent variables were coded as dummies (e.g. $0=$ No Emotions and $1=$ Emotions).

\section{RESULTS}

\section{Participants}

A total of 103 consultations between PNs $(n=19)$ and patients $(n=103)$ were analysed. All nineteen PNs were female with a mean age of 42 years (SD: 5.6). Patients' characteristics and their lifestyle behaviour are described within their SOC in Table 1. Only one significant difference was found between the patients' SOC: more patients are still in the (pre)contemplation or preparation phase for smoking behaviour.

\section{[TABLE 1]}

At the moment of the video-recorded consultation 45.7 percent of all patients were in the precontemplation or contemplation stage, 21.4 percent were in the preparation stage and 32.9 percent were in the action or maintenance stage. The significant correlations between the different SOC are medium to strong (range -0.37 to -0.64 ).

\section{PNs' generic communication skills, within the patient's stage of change}

Multilevel analyses (model 1) revealed a significant association with one communication skill measured using the MAAS-global: 'summarizations' (Table 2). PNs summarize less during their conversations with patients who are in the action or maintenance stage than with patients in the other stages. Furthermore, trends appear for the items 'request for help', 'management' and 'empathy'. PNs tend to pay more attention to 'request for help' and 'management' with patients in the preparation stage than with patients in other stages ( $\mathrm{P}=0.07$ and $\mathrm{P}=0.06$, respectively) and tend to show more empathy during the consultations with patient in the action or maintenance stage $(\mathrm{P}=0.07)$. 
Noordman, J., Vet, E. de, Weijden, T. van der, Dulmen, S. van. Motivational interviewing within the different stages of change: an analysis of practice nurse-patient consultations aimed at promoting a healthier lifestyle. Social Science \& Medicine: 2013, 87, 60-67

\section{[TABLE 2]}

The regression coefficients range from -0.73 to 1.60 . Hence, differences between the SOC are small. The intraclass correlation (ICC) on the generic communication skills range from $>0.01$ to 0.37 for model 1 . After correcting for patient characteristics and lifestyle behaviour (model 2) the association between 'summarizations' and the action and maintenance stage was no longer significant. The coefficients hardly changed after this correction (results not shown).

The item 'exploration' correlates significantly, on a medium level, with 'management', 'information giving' and 'structuring' (all Pearson's correlation of 0.41). Furthermore, the item 'structuring' also correlates significantly with 'empathy' (0.39). All other correlations are not significant or very small.

\section{PNs' clinical competence, within the patient's stage of change}

There were no items that showed significant different scores between the SOC with respect to the clinical competence of PNs (MAAS-global items 14-17) (Table 2). The intraclass correlation (ICC) on clinical competence range from 0.04 to 0.15 for model 1.

The clinical aspect 'diagnosis' correlates significantly with the clinical aspects 'history taking' (0.43) and 'management' (0.57).

\section{Motivational interview skills of PNs, within the patient's stage of change}

The majority of the BECCI-domains and separate BECCI items did not differ significantly between the three SOC. There were three BECCI items (items 1, 4 and 8) that did show significant differences between patient's SOC. PNs encourage patients to talk about behaviour change (item 4) more in the precontemplation and in the contemplation stage, than in the preparation stage and in the action and maintenance stage, and also more in the preparation stage than in the action and maintenance stage (see Table 3). This finding confirms our first hypothesis. PNs invite patients more often in the preparation stage to talk about behaviour change (item 1) than during the other stages. PNs 'acknowledge challenges about behaviour change that patients face' (item 8) less during the action and the maintenance stage than during the other SOC. These findings are fairly in line with hypotheses 2 and 4. The significant coefficients range from -0.43 to 0.59 . This indicates that differences between the SOC are small. The intraclass correlation (ICC) on the four BECCI domains and BECCI mean sum score range from 0.02 to 0.10 for model 1 . The ICC on the eleven BECCI items ranges from 0.02 to 0.25 for model 1 .

\section{[TABLE 3]}

The significant correlations between the four BECCI domains are between 0.38 and 0.5 , indicating medium correlations. The significant correlations between the items of the BECCI were small to medium and ranged from 0.21 to 0.53 . However, items six and seven of the BECCI show only significant (small) correlations with, respectively, item four and items four and five. 
Noordman, J., Vet, E. de, Weijden, T. van der, Dulmen, S. van. Motivational interviewing within the different stages of change: an analysis of practice nurse-patient consultations aimed at promoting a healthier lifestyle. Social Science \& Medicine: 2013, 87, 60-67

After correcting for patient characteristics and lifestyle behaviour most differences remain with the exception of 'PN acknowledges challenges about behaviour change that the patient faces' (item 8), which was only borderline significant. The (significant) coefficients hardly changed after this correction. However, these analyses reveal another positive significant association between the mean sum scores of the BECCI domains and the different SOC (results not shown). Apparently, PNs apply their MI skills on average more when patients are in the preparation stage than in the other SOC.

\section{Discussion}

The present study provides a first foray into the application of PNs' generic communication skills, clinical competence and MI skills within the patient's SOC, during actual consultations between patients and PNs. Our results showed that PNs do adjust their MI skills to patients' SOC, but only to some extent. PNs tailored one generic communication skill ('summarizations') to the patient's SOC, and trends did appear for the items 'request for help', 'management' and 'empathy'. Clinical competence, i.e. the application of evidence-based guidelines, were not adapted to patient's SOC.

Our findings did partly confirm our hypotheses. In particular - as expected - PNs do encourage patients to talk about behaviour change more in the precontemplation and contemplation stage than during the other stages, and also more during the preparation stage than during the action and maintenance stage. However, in contrast to what we expected, PNs invite the patient to talk about behaviour change in the preparation stage more than during the precontemplation and contemplation stage. Although PNs do invite the patient to talk about behaviour change more during the preparation stage than during the action and maintenance stage. The other hypotheses could not be confirmed, these being: that PNs are more likely 'to ask questions to elicit how the patient thinks and feels about behaviour change' during the precontemplation and contemplation stage than during the other stages; that PNs 'acknowledges challenges about behaviour change that the patient faces' less during the action and maintenance stage than during the other stages of change; that PNs and patients are more likely 'to exchange ideas about how the patient could change current behaviour' during the preparation stage than during the other SOC. PNs therefore apply their MI skills on average more when patients are in the preparation stage than in the other SOC. This suggests that, to some extent, PNs intuitively assess the stage of patients' readiness to change and tailor their communication consequently. After all, PNs (and patients) were not aware of the focus of our observations (i.e. patient's SOC), and in the end differences between the stages were small. However, PNs were aware that their MI skills were being evaluated.

As expected, PNs' clinical competence did not vary by SOC group. This finding supports the validity of our analyses; we were able to find (small) skill differences by SOC group for some MI and generic communication skills, but not for all skills (i.e. 
Noordman, J., Vet, E. de, Weijden, T. van der, Dulmen, S. van. Motivational interviewing within the different stages of change: an analysis of practice nurse-patient consultations aimed at promoting a healthier lifestyle. Social Science \& Medicine: 2013, 87, 60-67

clinical competence, among others). However, more research is needed to replicate these findings with larger groups of patients and in different settings.

Previous research suggests that interventions that are tailored to a person's SOC are better than generic approaches (Johnson et al., 2008; Prochaska, 1996; Ruggiero, 2000). A recent study on preventing weight gain also concludes that the stage to which a person belongs does matter (Ter Bogt, 2011). In contrast,Aveyard, Massey, Parsons, Manaseki, and Griffin (2009) reported that their stage-matched self-help intervention was not more effective for smoking cessation than their self-help control intervention. However, a commentary by Prochaska (2009) revealed that the control intervention in the study by Aveyard et al. (2009) was in fact also stage appropriate. Furthermore, earlier studies recommend using MI within the SOC Model, since it systematically guides patients towards motivation for change (Botelho \& Skinner, 1995; Elder et al., 1999). By teaching PNs to explicitly identify a patient's SOC they could enhance their adaptation of MI (and general communication) skills. This is especially pertinent since previous research (Noordman et al., 2012) suggests that MI skills are difficult to apply in daily practice.

It would thus appear that PNs amount of SOC tailoring in real-life consultations is small and could benefit from brief, reliable and valid screening instruments. For this purpose, Prochaska and colleagues have produced several questionnaires (available on their websitehttp://www.uri.edu/research/cprc/measures.html, accessed 18-012012) that can be used in daily practice to assess a patient's readiness of change.

\section{Strengths and limitations}

The present study provides an initial investigation into the daily application of PNs' MI and general communication skills to patients' SOC. Furthermore, we observed the BECCI independently of SOC (i.e. by using different observers) so that ratings of PNs' MI skills were not influenced by SOC scores. We also analysed the separate items of the BECCI instead of solely relying on the BECCI domain scores. Low Cronbachs alphascores for the BECCI domains justify this choice. In addition, SOC is a theoretical construct that can be easily and reliably used in daily practice because of its concreteness. By using SOC, healthcare providers can assess the level of a patient's involvement and readiness to change their behaviour, and evaluate the intervention, in contrast to other theories or models like the Health Belief Model and Social Cognitive theory (Elder et al., 1999). Besides, MI and SOC are complementary clinical theoretical frameworks. Another strength of our study is the high average interrater agreement scores between observers and the use of validated clinical measures to assess PN's skills (i.e. BECCI and MAAS-global). Some limitations should also be noted. Firstly, both observation protocols (the BECCI and the MAAS-global) focus on communication skills of healthcare providers and do not include patients' statements or the patient-provider interaction. Secondly, we used the MAAS-global for coding nurses' communication skills even though this instrument was originally developed for physicians. Therefore, some aspects of the protocol seem less relevant for observing PNs' communication. Since most patients visit the PN every three months over a lengthy period of time, the PN is already familiar with the patient's reason to attend the consultation. In this context, the item 'introduction' on the MAAS-global seems less applicable. In addition, the assessment of patient's SOC was based on the conversation between the patient and PN. We did not ask the patients themselves about their current SOC. Although the 
Noordman, J., Vet, E. de, Weijden, T. van der, Dulmen, S. van. Motivational interviewing within the different stages of change: an analysis of practice nurse-patient consultations aimed at promoting a healthier lifestyle. Social Science \& Medicine: 2013, 87, 60-67

independent coding of patient's SOC resulted in complete interrater agreement, future research should investigate the overlap between both methods.

We also grouped the stages of change together for analyses purposes, resulting in three combined stages instead of the five stages developed by Prochaska and DiClemente (1983). Although this could have affected our outcomes, our grouping was consistent with previous studies on SOC (De Vet, de Nooijer, de Vries, \& Brug, 2005; De Vet, de Nooijer, de Vries, \& Brug, 2008).

It was also not possible to observe all the elements of the Transtheoretical Model.

Therefore we selected the elements of 'consciousness raising' and 'dramatic relief', which were approximately in accordance with our hypotheses. We acknowledge that the Transtheoretical model also proposes other processes of change that are relevant in patient-provider interactions (Lange \& Tigges, 2005; Petrocelli, 2002; Prochaska et al., 1992). Future research should aim to observe other theoretical constructs of the Transtheoretical Model as well.

In addition, when a patient presented multiple unhealthy behaviours during one consultation we selected one behaviour for which we defined the SOC; (1) the behaviour for which SOC was clearly manifested during the consultation, and if still multiple behaviours remained (2) the behaviour for which acting upon change was, at that moment, most profitable (e.g. the earliest SOC), as indicated during the consultation. We gave priority to the (pre)contemplation and preparation stages over action and maintenance stages. It might well be that for some patients actually more than one behaviour needed to be addressed. It remains an empirical question whether multiple unhealthy behaviours should be targeted simultaneously or subsequently after one behaviour has been changed successfully (Prochaska, Spring, \& Nigg, 2008).

Finally, by selecting patients who smoked or did not adhere to the Dutch physical activity norm we could have underestimated the discussion of smoking and physical activity in other consultations between PNs and patients.

In conclusion, this is a first exploratory study examining how motivational interviewing (MI) is adapted to patients' stages of change (SOC). Further research is necessary to detect if similar results can be found among other populations, behaviours and countries. Future research could investigate if combining MI (and general communication skills) with SOC is the most (clinical) effective strategy for PNs, and other health care providers within primary care, in helping patients to change their lifestyle behaviour and also maintain healthy behaviour. This is even more important as a meta-analysis on MI by Miller's research group (Hettema, Steele, \& Miller, 2005) suggests that standard MI only may not suffice in producing longer-lasting effects. This can be done by comparing counselling that combines MI and SOC with counselling that is only based on MI.

\section{ETHICS COMMITTEE}

The study was carried out according to Dutch privacy legislation. The privacy regulations were approved by the Dutch Data Protection Authority. According to Dutch legislation, approval by a medical ethics committee was not required for this observational study. 
Noordman, J., Vet, E. de, Weijden, T. van der, Dulmen, S. van. Motivational interviewing within the different stages of change: an analysis of practice nurse-patient consultations aimed at promoting a healthier lifestyle. Social Science \& Medicine: 2013, 87, 60-67

\section{COMPETING INTERESTS}

The authors have stated that there are none.

\section{ACKNOWLEDGEMENTS}

We would like to thank the patients and PNs for their participation. We also thank Inge van der Lee and Nienke Franse for their help in data acquisition and coding of the video-recorded consultations. This study was funded by the Dutch Ministry of Health, Welfare and Sport. They had no role in the collection, analysis, interpretation, writing or decision to submit this article.

\section{APPENDiX 1.}

\section{MAAS-GLOBAL}

JACQUES VAN THIEL, PAUl RAM, JAN VAN DALEN, MAASTRICHT UNIVERSITY, NETHERLANDS 2000.

$0=$ not present, 1 = poor, 2 = unsatisfactory, $3=$ doubtful, $4=$ satisfactory, $5=$ good, $6=$ excellent, n.a. $=$ not applicable

\section{SECTION 1: COMMUNICATION SKILLS FOR EACH SEPARATE PHASE}

Introduction 0123456

giving the patient room to tell his story general orientation on the reason for visit asking about other reasons for visit

Follow-up consultation n.a. 0123456 naming previous complaints, requests for help and management plan asking about adherence to management plan asking about the course of the complaint

Request for help 0123456

naming requests for help, wishes or expectations naming reasons that prompted the patient to come now completing exploring request for help

Physical examination n.a. 0123456 instructions to the patient explanation of what is being done treating the patient with care and respect

Diagnosis 0123456

naming findings and diagnosis/hypothesis naming causes or the relation between findings and diagnosis naming prognosis or expected course asking for patient's response

Management 0123456 
Noordman, J., Vet, E. de, Weijden, T. van der, Dulmen, S. van. Motivational interviewing within the different stages of change: an analysis of practice nurse-patient consultations aimed at promoting a healthier lifestyle. Social Science \& Medicine: 2013, 87, 60-67

shared decision-making, discussing alternatives, risks and benefits

discussing feasibility and adherence

determining who will do what and when

asking for patient's response

Evaluation of consultation 0123456

general question

responding to requests for help

perspective for the time being

\section{SECTION 2: GENERAL COMMUNICATION SKILLS}

1. .Exploration 0123456

exploring requests for help, wishes or expectations

exploring patient's response to information given

within patient's frame of reference

responding to nonverbal behaviour and cues

\section{2. .Emotions 0123456}

asking about/exploring feelings

reflecting feelings (including nature and intensity)

sufficiently throughout the entire consultation

3. .Information giving 0123456

announcing, categorizing

in small quantities, concrete explanations

understandable language

asking whether the patient understands

4. .Summarizations 0123456

content is correct, complete

concise, rephrased

checking

sufficiently throughout the entire consultation

5. . Structuring 0123456

logical sequence of phases

balanced division of time

announcing (history taking,

examination, other phases)

6. .Empathy 0123456

concerned, inviting and sincerely empathetic in intonation, gesture and eye contact expressing empathy in brief verbal responses

\section{SECTION 3: MEDICAL ASPECTS}

Rate according to professional guidelines if they are available.

Otherwise rate to the best of your ability.

7. . History taking 0123456

8. . Physical examination n.a. 0123456 
Noordman, J., Vet, E. de, Weijden, T. van der, Dulmen, S. van. Motivational interviewing within the different stages of change: an analysis of practice nurse-patient consultations aimed at promoting a healthier lifestyle. Social Science \& Medicine: 2013, 87, 60-67

9. . Diagnosis 0123456

10. . Management 0123456

Other feedback

APPENDIX 2.

\section{The Behaviour Change Counselling Index (BECCI)}

\section{Domain 1: Agenda setting and permission seeking}

1. The practitioner invites the patient to talk about behaviour change.

2. The practitioner demonstrates sensitivity to talking about other issues.

\section{DOMAIN 2: THE WHY AND HOW OF CHANGE IN BEHAVIOUR}

3. The practitioner encourages the patient to talk about current behaviour or status quo.

4. The practitioner encourages the patient to talk about behaviour change.

5. The practitioner asks questions to elicit how the patient thinks and feels about the topic.

6. The practitioner uses empathic statements when the patient talks about the topic.

7. The practitioner uses summaries to bring together what the patient says about the topic.

\section{DOMAIN 3: THE WHOLE CONSULTATION}

8. The practitioner acknowledges challenges about behaviour change that the patient faces.

9. .When the practitioner provides information, it is sensitive to the patient's concerns and understanding.

10. 0 .The practitioner actively conveys respect for the patient's choice about behaviour change.

\section{DOMAIN 4: TALK ABOUT TARGETS}

11. The practitioner and the patient exchange ideas about how the patient could change current behaviour.

$0=$ Not at all, $1=$ Minimally, $2=$ To some extent, $3=$ A good deal, $4=$ A great extent

\section{MEAN SUBSTITUTION:}

11. . Take the mean of all the applicable items (i.e. add up the total score of the applicable items, and divide by that number of items). 
Noordman, J., Vet, E. de, Weijden, T. van der, Dulmen, S. van. Motivational interviewing within the different stages of change: an analysis of practice nurse-patient consultations aimed at promoting a healthier lifestyle. Social Science \& Medicine: 2013, 87, 60-67

12. A technique known as 'mean substitution' is used for any items scored as 'not applicable'. The mean of the applicable items in a particular consultation is the score to be used for the not applicable items. So, for example, if the mean of all other items is 2.87, this is the score that should be given to any items scored as not applicable (Lane, 2002; Lane et al., 2005).

\section{REFERENCES}

1. Aveyard et al., 2009 P. Aveyard, L. Massey, A. Parsons, S. Manaseki, C. Griffin The effect of transtheoretical model based interventions on smoking cessation Social Science \& Medicine, 68 (2009), pp. 397-403

2. Botelho and Skinner, 1995 R.J. Botelho, H. Skinner Motivating change in health behavior: implications for health promotion and disease prevention Primary Care, 22 (1995), pp. 565-589

3. Bowen et al., 2002 D. Bowen, C. Ehret, M. Pederson, L. Snetselaar, M. Johnson, L. Tinker et al. Results of an adjunct dietary intervention program in the Women's Health Initiative Journal of the American Dietetic Association, 102 (11) (2002), pp. 1631-1637

4. Britt et al., $2004 \mathrm{E}$. Britt, S. Hudson, N. Blampied Motivational interviewing in health settings: a review Patient Education \& Counseling, 53 (2004), pp. 147-155

5. Coups et al., 2004 E.J. Coups, A. Gaba, C.T. Orleans Physician screening for multiple behavioral health risk factors American Journal of Preventive Medicine, 27

(2) (2004), pp. 34-41

6. Davis et al., 2011 M.F. Davis, D. Shapiro, R. Windsor, P. Whalen, R. Rhode, H.S. Miller et al. Motivational interviewing versus prescriptive advice for smokers who are not ready to quit Patient Education and Counseling, 83 (2011), pp. 129-133

7. De Vet et al., 2005 E. De Vet, J. de Nooijer, N.K. de Vries, J. Brug Determinants of forward stage transition from precontemplation and contemplation for fruit consumption American Journal of Health Promotion, 19 (2005), pp. 275-285

8. De Vet et al., 2008 E. De Vet, J. de Nooijer, N.K. de Vries, J. Brug Do the transtheoretical processes of change predict transitions in stages of change for fruit intake? Health Education \& Behavior, 35 (2008), pp. 603-618

9. Elder et al., 1999 J.P. Elder, G.X. Ayala, S. Harris Theories and intervention approaches to health-behavior change in primary care American Journal of Preventive Medicine, 17 (4) (1999), pp. 275-284

10. Emmons and Rollnick, 2001 K.M. Emmons, S. Rollnick Motivational interviewing in health care settings. Opportunities and limitations American Journal of Preventive Medicine, 20 (1) (2001), pp. 68-74

11. Hardcastle et al., 2008 S. Hardcastle, A. Taylor, M. Bailey, R. Castle A randomized controlled trial on the effectiveness of a primary health care based counseling intervention on physical activity, diet and CHD risk factors Patient Education \& Counseling, 70 (1) (2008), pp. 31-39

12. Hettema et al., 2005 J. Hettema, J. Steele, W.R. Miller Motivational interviewing Annual Review of Clinical Psychology, 1 (2005), pp. 91-111

13. Johnson et al., 2008 S.S. Johnson, A.L. Paiva, C.O. Cummins, J.L. Johnson, S.J. Dyment, J.A. Wrightet al. Transtheoretical model-based multiple behavior 
Noordman, J., Vet, E. de, Weijden, T. van der, Dulmen, S. van. Motivational interviewing within the different stages of change: an analysis of practice nurse-patient consultations aimed at promoting a healthier lifestyle. Social Science \& Medicine: 2013, 87, 60-67

intervention for weight management: effectiveness on a population basis Preventive Medicine, 46 (2008), pp. 238-246

14. Kemper et al., 2000 H.G.C. Kemper, W.T.M. Ooijendijk, M. Stiggelbout Consensus over de Nederlandse Norm voor Gezond Bewegen Tijdschrift Voor Sociale

Gezondheidszorg, 78 (2000), pp. 180-183

15. Lane, $2002 \mathrm{C}$. Lane The behaviour change counselling Index (BECCI). Manual for coding behaviour change counseling (2002) http://motivationalinterview.net/library/BECCIManual.pdf Accessed 18.01.12

16. Lane et al., 2005 C. Lane, M. Huws-Thomas, K. Hood, S. Rollnick, K. Edwards, M. Robling Measuring adaptations of motivational interviewing: the development and validation of the behaviour change counselling index $(\mathrm{BECCl})$ Patient Education and Counseling, 56 (2005), pp. 166-173

17. Lange and Tigges, 2005 N. Lange, B.B. Tigges Influence positive change with motivational interviewing The Nurse Practitioner, 30 (3) (2005), pp. 44-53

18. Laurant et al., 2009 M. Laurant, M. Harmsen, H. Wollersheim, R. Grol, M. Faber, B. Sibbald The impact of nonphysician clinicians: do they improve the quality and costeffectiveness of health care services? Medical Care Research and Review, 66 (6) (2009), pp. 36s-89s

19. Martins and McNeil, 2009 R.K. Martins, D.W. McNeil Review of motivational interviewing in promoting health behaviors Clinical Psychology Review, 29 (4) (2009), pp. 283-293

20. Miller, 1996 W.R. Miller Motivational interviewing: research, practice and puzzles Addictive Behaviors, 21 (1996), pp. 835-842

21. Miller and Rollnick, 2002 W.R. Miller, S. Rollnick Motivational interviewing: Preparing people for change (2nd ed.)The Guilford Press, New York (2002)

22. Miller and Rollnick, 2004 W.R. Miller, S. Rollnick Talking oneself into change: motivational interviewing, stages of change, and therapeutic process Journal of Cognitive Psychotherapy: An International Quarterly, 18 (4) (2004), pp. 299-308

23. Moran et al., $2008 \mathrm{~J}$. Moran, H. Bekker, G. Latchford Everyday use of patientcentred, motivational techniques in routine consultations between doctors and patients with diabetes Patient Education and Counseling, 73 (2008), pp. 224-231

24. Nielen et al., 2010 .M.J. Nielen, W.J.J. Assendelft, A.J.M. Drenthen, P. van den Hombergh, I. van Dis, F.G. Schellevis Primary prevention of cardio-metabolic diseases in general practice: a Dutch survey of attitudes and working methods of general practitioners European Journal of General Practice, 16 (2010), pp. 139-142

25. NNGB, 1998 NNGB Nederlandse Norm Gezond Bewegen (1998) 18-012012 http://www.kiesbeter.nl/medische-informatie/leefwiize/bewegen/norm-gezondbewegen/

26. Noldus et al., 2000 L.P. Noldus, R.J. Trienes, A.H. Hendriksen, H. Jansen, R.G. Jansen The Observer-Video-Pro: new software for the collection, management and presentation of time-structured data from videotapes and digital media films Behavior Research Methods, Instruments, \& Computers, 32 (2000), pp. 197-206

27. Noordman et al., 2012 J. Noordman, I. van der Lee, M. Nielen, H. Vlek, T. van der Weijden, S. van Dulmen Do trained practice nurses apply motivational interviewing techniques in primary care consultations? Journal of Clinical Medicine Research, 4 (6) (2012), pp. 393-401

28. Noordman et al., 2010 J. Noordman, P. Verhaak, I. van Beljouw, S. van Dulmen Consulting room computers and their effect on GP-patient communication Family Practice, 27 (6) (2010), pp. 644-651 
Noordman, J., Vet, E. de, Weijden, T. van der, Dulmen, S. van. Motivational interviewing within/ the different stages of change: an analysis of practice nurse-patient consultations aimed at promoting a healthier lifestyle. Social Science \& Medicine: 2013, 87, 60-67

29. Petrocelli, 2002 J.V. Petrocelli Processes and stages of change: counseling with the transtheoretical model of change Journal of Counselling \& Development, 80 (2002), pp. 22-30

30. Prochaska, 1996 J.O. Prochaska A stage paradigm for integrating clinical and public health approaches to smoking cessation Addictive Behaviors, 21 (6) (1996), pp. 721-732 w

31. Prochaska, 2009 J.O. Prochaska Flaws in the theory or flaws in the study: a commentary on "The effect of Transtheoretical Model based interventions on smoking cessation" Social Science \& Medicine, 68 (2009), pp. 404-406

32. Prochaska and DiClemente, 1983 J.O. Prochaska, C.C. DiClemente Stages and processes of self-change of smoking: toward an integrative model of change Journal of Consulting and Clinical Psychology, 51 (3) (1983), pp. 390-395

33. Prochaska et al., 1992 J.O. Prochaska, C.C. DiClemente, J.C. Norcross In search of how people change. Applications to addictive behaviors American Psychologist, 47

(9) (1992), pp. 1102-1114

34. Prochaska et al., 2002 J.O. Prochaska, C.A. Redding, K. Evers The transtheoretical model and stages of change K. Glanz, B.K. Rimer, F.M. Lewis (Eds.), Health behavior and health education: Theory, research, and practice, Jossey-Bass, San Francisco, CA (2002), pp. 99-120

35. Prochaska et al., 2008 J.O. Prochaska, B. Spring, C.R. Nigg Multiple health behavior change research: an introduction and overview Preventive Medicine, 46 (3) (2008), pp. 181-188

36. Rabe-Hesketh et al., 2002 S. Rabe-Hesketh, A. Skrondal, A. Pickles Reliable estimation of generalized linear mixed models using adaptive quadrature The Stata Journal, 2 (1) (2002), pp. 1-21

37. Richards et al., 2000 A. Richards, J. Carley, S. Jenkins-Clarke, D.A. Richards Skill mix between nurses and doctors working in primary-care delegation or allocation: a review of the literature International Journal of Nursing Studies, 37 (2000), pp. 185197

38. Richards et al., 2006 A. Richards, K.K. Kattelmann, C. Ren Motivating 18- to 24year-olds to increase their fruit and vegetable consumption Journal of the American Dietetic Association, 106 (9) (2006), pp. 1405-1411

39. Ruggiero, 2000 L. Ruggiero Helping people with diabetes change behaviour: from theory to practice Diabetes Spectrum, 13 (3) (2000), p. 125

40. Sim and Wright, 2005 J. Sim, C.C. Wright The kappa statistic in reliability studies: use, interpretation, and sample size requirements Physical Therapy, 85 (3) (2005), pp. 257-268

41. Snijders and Bosker, 1999 T.A.B. Snijders, R.J. Bosker Multilevel analysis. An introduction to basic and advanced multilevel modeling (1st ed.)Sage, London (1999)

42. Spollen et al., 2010 J.J. Spollen, C.R. Thrush, D. Mui, M.B. Woods, S.G. Tariq, E. Hicks A randomized controlled trial of behaviour change counselling education for medical students Medical Teacher, 32 (2010), pp. e170-e177

43. Stata, 2009 Stata [computer program] (2009). Version 11. Copyright 2009 by StataCorp LP, 4905 Lakeway Drive, College Station, TX 77845 USA. www.stata.com.

44. Ter Bogt, 2011 N.C.W. Ter Bogt Preventing weight gain in general practice: Lifestyle counselling by nurse practitioners compared with usual care by general practitioners $\mathrm{PhD}$ thesis Rijksuniversiteit Groningen, Groningen (2011)http://irs.ub.rug.nl.proxy.library.uu.nl/ppn/332486931 Accessed 18.01.12 
Noordman, J., Vet, E. de, Weijden, T. van der, Dulmen, S. van. Motivational interviewing within the different stages of change: an analysis of practice nurse-patient consultations aimed at promoting a healthier lifestyle. Social Science \& Medicine: 2013, 87, 60-67

45. Van Thiel et al., 2000 J. Van Thiel, P. Ram, J. van Dalen MAAS-global manual Maastricht University, Maastricht (2000) http://www.hag.unimaas.nl/MaasGlobal_2000/GB/MAAS-Global-2000-EN.pdf Accessed 18.01.12

46. West, 2005 R. West Time for a change: putting the Transtheoretical (Stages of Change) Model to rest Addiction, 100 (2005), pp. 1036-1039

47. WHO, $2002 \mathrm{WHO}$ Innovative care for chronic conditions: Building blocks for action WHO, Geneva (2002) 
Table 1

Characteristics of patients ( $n=103$ ) within their (observed) stage of change.

\begin{tabular}{|c|c|c|c|c|}
\hline & $\begin{array}{l}\text { Patients in the pre-contemplation } \\
\text { or contemplation stage } n=47\end{array}$ & $\begin{array}{l}\text { Patients in the preparation } \\
\text { stage } n=22\end{array}$ & $\begin{array}{l}\text { Patients in the action or } \\
\text { maintenance stage } n=34\end{array}$ & P-value \\
\hline Mean age in years (SD) & $58.2(14.7)$ & $61.4(10.8)$ & $61.8(12.6)$ & 0.41 \\
\hline Men (\%) & 64 & 41 & 44 & 0.10 \\
\hline Educational level (\%) low & 29.6 & 35.0 & 303 & 096 \\
\hline middle & 56.8 & 45.0 & 48.5 & \\
\hline high & 13.6 & 20.0 & 21.2 & \\
\hline Married/living together $(\%)$ & 76 & 71 & 73 & 0.84 \\
\hline Dutch ethnicity (\%) & 85 & 82 & 79 & 0.53 \\
\hline $\begin{array}{l}\text { Smoking; daily/now and then, } \\
\text { according to patients (\%) }\end{array}$ & 51 & 41 & 24 & $0.04^{2}$ \\
\hline $\begin{array}{l}\text { Meets recommended physical } \\
\text { activity level, according to patients }(\%)^{\mathrm{b}}\end{array}$ & 52 & 52 & 41 & 0.58 \\
\hline Mean consultation length (minutes) & 24.5 & 23.9 & 22.7 & 0.45 \\
\hline Disease/risk factor (\%): & & & & 0.41 \\
\hline Diabetes type 2 & 44.7 & 40.9 & 58.8 & \\
\hline COPD/asthma & 17.0 & 13.6 & 5.9 & \\
\hline Heart \& vascular disease & 4.3 & - & - & \\
\hline Hypertension & 25.5 & 27.3 & 23.5 & \\
\hline Other & 8.5 & 18.2 & 11.8 & \\
\hline
\end{tabular}

a Significant difference between the stages of change, $x^{2}(P<0.05)$.

b Patient's (un) healthy lifestyle behaviour does not always correspond with the observed stage of change. It is possible that a patient who meets the recommended level of physical activity (action or maintenance stage) also smokes and has no intention to change this smoking behaviour, and is therefore selected as a patient in the precontemplation stage 
Table 2

Regression coefficients of the stages of change on the MAAS-global items (Model 1).

\begin{tabular}{|c|c|c|c|}
\hline & $\begin{array}{l}\text { Stage of change: Pre-\&contemplation } \\
\text { (regression coefficient, } 95 \% \mathrm{Cl} \text { ) }\end{array}$ & $\begin{array}{l}\text { Stage of change: Preparation } \\
\text { (regression coefficient, 95\% Cl) }\end{array}$ & $\begin{array}{l}\text { Stage of change: action \& maintenance } \\
\text { (regression coefficient, } 95 \% \mathrm{Cl} \text { ) }\end{array}$ \\
\hline \multicolumn{4}{|c|}{ MAAS-global } \\
\hline \multicolumn{4}{|c|}{ Section 1: Communication skills for each separate phase } \\
\hline 1. Introduction & $0.84(-0.20$ to 1.88$)$ & $-0.61(-1.66$ to 0.43$)$ & $-0.38(-1.44$ to 0.68$)$ \\
\hline 2. Follow-up consultation & $-0.32(-0.79$ to 0.16$)$ & $-0.03(-0.64$ to 0.58$)$ & $0.39(-0.11$ to 0.89$)$ \\
\hline 3. Request for help & $-0.43(-1.23$ to 0.38$)$ & $0.97(-0.08$ to 2.01$)$ & $-0.22(-1.07$ to 0.63$)$ \\
\hline 4. Physical examination & $0.06(-0.39$ to 0.51$)$ & $-0.02(-0.60$ to 0.56$)$ & $-0.05(-0.54$ to 0.43$)$ \\
\hline 5. Diagnosis & $0.03(-0.39$ to 0.45$)$ & $0.21(-0.29$ to 0.72$)$ & $-0.20(-0.64$ to 0.24$)$ \\
\hline 6. Management & $0.22(-0.14$ to 0.58$)$ & $0.14(-0.31$ to 0.58$)$ & $-0.37(-0.76$ to 0.01$)$ \\
\hline 7. Evaluation of consultation & $0.17(-0.76$ to 1.11$)$ & $-0.42(-1.56$ to 0.72$)$ & $0.13(-0.89$ to 1.14$)$ \\
\hline \multicolumn{4}{|c|}{ Section 2: General communication skills } \\
\hline 8. Exploration & $0.01(-0.29$ to 0.31$)$ & $0.32(-0.04$ to 0.68$)$ & $-0.27(-0.59$ to 0.04$)$ \\
\hline 9. Emotions & $-0.53(-1.43$ to 0.38$)$ & $-0.04(-1.15$ to 1.06$)$ & $0.70(-0.33$ to 1.74$)$ \\
\hline 10. Information giving & $0.12(-0.18$ to 0.42$)$ & $0.01(-0.36$ to 0.38$)$ & $-0.15(-0.47$ to 0.17$)$ \\
\hline 11. Summarizations & $0.32(-0.31$ to 0.95$]$ & $0.37(-0.40$ to 1.14$)$ & $-0.65^{\mathrm{a}}(-1.32$ to -0.01$)$ \\
\hline 12. Structuring & $-0.02(-0.29$ to 0.25$)$ & $0.29(-0.04$ to 0.62$)$ & $-0.20(-0.50$ to 0.09$)$ \\
\hline 13. Empathy & $-0.09(-0.27$ to 0.10$)$ & $-0.11(-0.33$ to 0.12$)$ & $0.18(-0.02$ to 0.37$)$ \\
\hline \multicolumn{4}{|c|}{ Section 3: Medical aspects (comply with guidelines) } \\
\hline 14. History taking & $0.07(-0.31$ to 0.46$]$ & $0.07(-0.40$ to 0.55$)$ & $-0.15(-0.57$ to 0.27$)$ \\
\hline 15. Physical examination & $0.01(-0.19$ to 0.21$)$ & $0.04(-0.21$ to 0.30$)$ & $-0.04(-0.25$ to 0.17$)$ \\
\hline 16. Diagnosis & $0.07(-0.15$ to 0.30$)$ & $0.15(-0.12$ to 0.42$)$ & $-0.21(-0.44$ to 0.03$)$ \\
\hline 17. Management & $0.03(-0.22$ to 0.28$]$ & $0.07(-0.24$ to 0.38$)$ & $-0.09(-0.36$ to 0.18$)$ \\
\hline
\end{tabular}

a Significant difference between this stage of change and the other stages of change on MAAS-global item $(P<0.05)$. 